\title{
Revisão bibliográfica sobre o fluxo de informações do processo de cuidados nutricionais (PCN) de dietas orais em hospitais: demanda por estudos em design da informação
}

\author{
Literature review on the information flow of the nutritional care process (PCN) of \\ oral diets in hospitals: demand for studies in information design
}

\author{
Grace Maria Cavalcanti Sampaio, Carla Galvão Spinillo
}

design da informação, processo de cuidados nutricionais, fluxo de informações, dietas orais em hospitais.

Este artigo apresenta uma revisão sistemática de literatura com base no modelo de Levi \& Ellis (2006), referente às áreas relacionadas à assistência nutricional em hospitais, processo e fluxo de informações mediante o uso de artefatos gráficos. O intuito desta revisão bibliográfica foi pontuar campos de conhecimento no qual o design da informação possa contribuir, minimizando problemas de comunicação que possam conduzir a incidentes na administração de serviços nutricionais. Os resultados revelam uma escassez de publicações, nacionais e internacionais, sobre o design da informação no âmbito da saúde, especificamente no campo da nutrição hospitalar, que estejam indexados nas principais bases de dados. Por fim são apresentadas as dificuldades encontradas no desenvolvimento da pesquisa.

Information design, nutritional care process, information flow, oral diets in hospitals.

This article presents a systematic literature review based on the guide produced by Levi and Ellis (2006), referring to areas related to nutritional assistance in hospitals, process and information flow through the use of graphic artifacts. The purpose of this literature review was to point out fields of knowledge in which information design can contribute, minimizing communication problems that could lead to incidents during the management of nutritional services. The results show the scarcity of national and international publications on Information design for the healthcare industry, specifically in the field of hospital nutrition, which are indexed in the main databases. The challenges faced during the development of the research are shown at the end of this review.

\section{Introdução}

Em hospitais, artefatos gráficos ${ }^{1}$ participam de forma rotineira como suporte para o registro e disseminação de informações e, portanto, são essenciais para a comunicação entre os profissionais que lá atuam. Isto é sobretudo importante no processo que envolve os

\footnotetext{
${ }^{1}$ Esta pesquisa adota a definição de artefatos gráficos como suportes mediadores para o registro, armazenamento, visualização e recuperação da informação, com base na definição de artefatos apresentada por Ninin (2009, p. 353): "Artefatos são todos os meios que os sujeitos têm à sua disposição para influenciar o objeto da atividade e transformálo em resultado. [...] Exercem o papel de mediadores entre os elementos de uma atividade."
}

Anais do $10^{\circ} \mathrm{CIDI}$ e $10^{\circ} \mathrm{CONGIC}$

Kelli C.A.S. Smythe, Rafael de Castro Andrade (orgs.)

Sociedade Brasileira de Design da Informação - SBDI

Curitiba | Brasil | 2021
Proceedings of the $10^{\text {th }} \mathrm{CIDI}$ and $10^{\text {th }}$ CONGIC

Kelli C.A.S. Smythe, Rafael de Castro Andrade (orgs.)

Sociedade Brasileira de Design da Informação - SBDI Curitiba | Brazil | 2021 
procedimentos para o atendimento da nutrição hospitalar. As informações que participam do atendimento da nutrição hospitalar são registradas em documentos impressos e/ou digitais (Cunha \& Silva, 2005; Hakel-Smith \& Lewis, 2004), além de quadros fixados em paredes ou disponíveis no modo digital. Nesse âmbito, os artefatos gráficos (e.g. formulários, quadros, slides) regulam as atividades, atuam como suporte para a descrição de tarefas prescritas, estabelecem a sequência para a execução das operações, além de contribuírem para a delegação de responsabilidades (Peruzzi, 2019; Brito, 2015). Neste ambiente, os artefatos gráficos são o veículo de informação que integram e apoiam essas demandas.

No processo relativo ao atendimento da nutrição hospitalar, as informações necessárias para a viabilização das atividades, são distribuídas nos ambientes e entre os profissionais, por meio do fluxo informacional, e opera como principal recurso para tomadas de decisão (Ceriolli et al., 2015; Greef et al., 2012). No âmbito da nutrição hospitalar, o processo está relacionado a investigação, consideração, planejamento e implementação do cuidado nutricional de um indivíduo, através da: avaliação do estado nutricional, identificação das necessidades ou problemas nutricionais e tratamento (American Dietetic Association, 1994; Pedroso et al., 2011). A este processo atribui-se a versão traduzida para o português da nomenclatura Nutricional Care Process, ou seja, Processo de Cuidados Nutricionais (PCN), já utilizada por outros pesquisadores (De Seta et al., 2010; Pedroso, 2007).

Em hospitais, este processo ocorre de forma contínua, envolvendo diversos profissionais de saúde (médicos, nutricionistas, enfermeiros, etc.), profissionais do setor de produção (cozinheiro, auxiliar, copeira, etc.), o paciente e seus acompanhantes. Logo, inicia com a avaliação nutricional (triagem), no momento da admissão no hospital (Mowe et al., 2006) e prossegue durante a estadia. Assim, a assistência nutricional em hospitais requer atenção e gerenciamento de diferentes atividades e registro em documentos com o objetivo de garantir que os pacientes recebam o suporte de nutrientes necessários e corretos (Howard et al., 2006), mediante a indicação da dieta, como medida primordial para a evolução clínica. Dessa forma, o trabalho da equipe de profissionais precisa ser sistematizado quanto aos procedimentos relativos a assistência nutricional, as rotinas que proporcionam a padronização das ações, os registros, de modo a obter o acompanhamento e controle do processo (Maculevicius \& Dias, 2006).

Nesse sentido, Howard et al. (2006) pontuam que as etapas do PCN: triagem, avaliação e tratamento devem ser monitoradas e documentadas. Em vista disso, artefatos gráficos exercem um papel relevante como suporte para o registro, armazenamento, visualização e continuidade das informações, necessária ao fluxo do processo.

Assim, para que a apresentação da informação possa contribuir para viabilização e o trânsito das atividades diárias é preciso compreensão do trabalho interdisciplinar, o conhecimento da sequência de procedimentos e os tipos de dados e informações que circulam nessas atividades (Sless, 1996). Destaca-se que, no planejamento e implementação do processo de cuidados nutricionais, os artefatos gráficos participam como base e estrutura para o registro e veiculação de informações para a realização e atendimento de inúmeras atividades. 
Mas, quando os profissionais de saúde utilizam esses artefatos (e.g. formulários) alguns autores relatam falhas resultantes do registro de informações. Quando a informação é registrada à mão há relatos de problemas referentes a caligrafia (letra manuscrita), presença de rasuras, além de formulários onde não há o registro de informações (Fontenele et al., 2019; Pena \& Melleiro, 2017; Quitério et al., 2016). Quando a informação é registrada em suportes digitais, a literatura aponta para prescrição de medicamentos, prescrição dietética (Porterfield et al., 2014; Valadão et al., 2009), e enfatiza que o registro de forma digital eliminam as dificuldades na compreensão da informação ocasionados pelo preenchimento à mão. Porém, mesmo tendo acesso a sistemas digitais, alguns profissionais de saúde registram as atividades manualmente (Reis et al., 2004), reiterando problemas anteriormente mencionados.

Diante da possibilidade do registro de informações ocorrer com falhas, considera-se que o design da informação pode atribuir valor, tornando a informação mais acessível, confiável, relevante e clara (Audy et al., 2005), além de favorecer à resolução de problemas relativos aos artefatos gráficos, possibilitando que o trabalho ocorra com fluência.

Assim, aspectos e princípios do design da informação como por exemplo: distribuição equilibrada de espaços para a inserção de informações/dados, estabelecimento de hierarquias e ênfases aplicadas aos grupos de informações (Pettersson, 2012b; Waarde, 2004; Horn, 1998; Schriver, 1997), entre outros, podem orientar e facilitar tanto o preenchimento desses artefatos, como a localização de dados.

Em vista disso, para vislumbrar como o design de informação pode contribuir na documentação de serviços clínicos em nutrição hospitalar é preciso, primeiramente, compreender o processo e os meios utilizados para documentar as informações acerca do estado de saúde e das prescrições do paciente (Hakel-Smith, 2004).

Para tanto faz-se necessário pesquisar e apreender os campos de estudo relacionados a assistência nutricional, processo, fluxo de informações em hospitais, assim como design da informação direcionado a artefatos gráficos que participem desse contexto. Assim, o intuito dessa revisão bibliográfica é pontuar campos de conhecimento no qual o design da informação possa contribuir, minimizando problemas de comunicação que possam conduzir a incidentes na administração de serviços nutricionais.

Diante dessa necessidade foi elaborada uma revisão bibliográfica sistemática, de modo a construir uma pesquisa com amplitude, profundidade e rigor, com base em etapas sistemáticas de atividades (Levi \& Ellis, 2006). O método utilizado para a realização desta revisão sistemática encontra-se na próxima seção.

\section{Método da revisão bibliográfica sistemática}

Diante das considerações apresentadas, e mediante o modelo descrito por Levi e Ellis (2006), surge a seguinte questão de pesquisa: "Como o design da informação pode contribuir para o fluxo de informações do processo de cuidados nutricionais (PCN) de dietas orais em hospitais? Mediante esse objetivo primário foram estabelecidas as questões secundárias da pesquisa: 
(1) Como ocorre o processo de cuidados nutricionais ( $P C N)$ para dietas orais em hospitais?

(2) Como ocorre o fluxo de informações no processo de prescrição de dietas orais em hospitais? (3) Como o design da informação participa do fluxo de informações no processo de prescrição de dietas orais em hospitais?

Para o desenvolvimento da pesquisa foi elaborado um protocolo (LEVI \& ELLIS, 2006) que descreve uma revisão bibliográfica por meio de uma abordagem "entrada-processamentosaída", considerando processo como uma sequência de passos e atividades. A figura 1 ilustra as três fases de uma RBS proposta pelos autores.

Figura 1: Três fases de uma RBS. Fonte: Adaptado de Levy e Ellis (2006).

\section{Entrada}

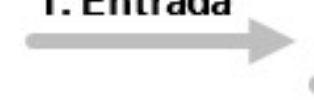

\section{Processamento}

- Conhecer a literatura

- Compreendera

literatura

- Aplicar a revisão

- Analisar resultados

- Síntese

- Avaliar os resultados

\section{Saída}

O modelo proposto por Levi e Ellis (2006) estabelece que a entrada se refira a qualidade da literatura que será pesquisada. Nesta fase da pesquisa foram delimitados: a equipe; os objetivos, as questões da pesquisa, os domínios da pesquisa, as palavras-chave, os strings (estratégias de busca) realizados conforme as palavras-chave e os critérios de inclusão, os idiomas, as bases e as revistas consideradas relevantes de cada área estabelecida na busca (nutrição, gestão da informação e design), os critérios de inclusão e exclusão (filtros), e por fim os critérios de qualidade. A segunda fase, ou seja, o processamento, deve ocorrer em ciclos conforme o conhecimento sobre o tema vai aumentando. Esse ciclo pode reiniciar quantas vezes o pesquisador julgue necessário, até que as respostas às questões da pesquisa sejam alcançadas. Neste ponto da pesquisa foram conduzidas as seguintes atividades: configuração das buscas avançadas em cada base/revista (filtros); buscas, exportação dos artigos encontrados, análise inicial dos resultados (leitura dos títulos, palavras-chave e resumos), aplicação dos critérios de inclusão e exclusão, e por fim a leitura completa dos que foram selecionados. Esta etapa foi revisada em ciclos durante dois meses em busca de novos artigos. Por último, a saída refere-se à terceira fase e compreende a síntese e a discussão dos resultados. A figura 2 descreve todas as etapas da revisão bibliográfica. 
Figura 2: Etapas do método da revisão bibliográfica. Fonte: Elaborado pelas autoras.

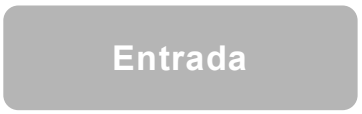

Equipe

Objetivos

Questões da pesquisa

Domínios da pesquisa

Palavras-chave

Strings de busca

Idiomas

Bases

Revistas

Período da busca

Critérios de inclusão

Critérios de exclusão

Critérios de qualidade

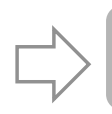

Configuração das

buscas avançadas

em cada base/revista

Condução das buscas

Exportação dos artigos

Análise inicial dos

resultados

Aplicação dos critérios

de inclusão e exclusão

Leitura completa dos

artigos selecionados

Avaliação dos resultados

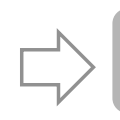

Saída

Síntese e discussão dos resultados

\section{Fase 1 da RBS: Entrada}

Para esta revisão foram planejadas buscas de artigos científicos publicados em anais de congressos indexados em bases de dados e no Banco de teses da Capes. Adicionalmente foram realizadas buscas em revistas especializadas nos campos de estudo relativos à assistência nutricional, processo e fluxo de informações em hospitais, design da informação, que não se encontram indexadas nas bases de dados. As bases de dados selecionadas para a busca foram: BVS; Lilacs; Scopus; Pubmed; Science Direct; Ebsco e Springer.

No campo da Nutrição foram pesquisadas as revistas: European Journal Of Clinical Nutrition - Nature; Demetra, Rasbran, e The American Journal of Clinical Nutrition (AJCN). Algumas revistas e portais foram descartados por já estarem indexados em algumas bases como: Clinical Nutrition Espen (indexada pela Science Direct), Revista Nutrire (indexada pela Springer), FSTA e CINAHL (vinculados a EBSCO).

No âmbito da Gestão da Informação foram pesquisadas as seguintes revistas: Revistas Eletrônicas Puc/SP; Revista Economia e Gestão Puc/MG; Revista Gestão Organizacional /Unochapecó; Navus - Revista de Gestão e Tecnologia SENAC/SC; Revista de Gestão em Sistemas de Saúde; Revista Eletrônica de Sistemas de Informação; Revista Eletrônica Gestão \& Saúde/UNB. As revistas REGE - Revista de Gestão USP (Ebsco) e a Revista de Gestão da Tecnologia e Sistemas de Informação (Scielo) foram descartados por já estarem indexadas em bases de dados.

Por fim, as revistas de design consideradas na pesquisa foram: Design e Tecnologia; Estudos em Design; Infodesign; Strategic Design Research Journal (Unisinos) e Information Design Journal (IDJ). 
A revisão bibliográfica foi conduzida entre os meses de março e abril de 2021. A busca por artigos compreendeu o período de 2011 a 2021, nos idiomas português e inglês. O quadro 1 apresenta esta primeira fase (entrada) da pesquisa com seu planejamento.

Quadro 1: Primeira fase da revisão bibliográfica (entrada). Fonte: Elaborado pelas autoras.

\begin{tabular}{|c|c|}
\hline Objetivo & $\begin{array}{l}\text { Pontuar campos de conhecimento em que o design da informação possa contribuir, } \\
\text { minimizando problemas de comunicação que possam conduzir a incidentes na } \\
\text { administração de serviços nutricionais em hospitais }\end{array}$ \\
\hline $\begin{array}{l}\text { Questão } \\
\text { da pesquisa }\end{array}$ & $\begin{array}{l}\text { Como o design da informação pode contribuir para o fluxo de informações do processo } \\
\text { de cuidados nutricionais (PCN) de dietas orais em hospitais? }\end{array}$ \\
\hline $\begin{array}{l}\text { Questões } \\
\text { secundárias }\end{array}$ & $\begin{array}{l}\text { (1) Como ocorre o processo de cuidados nutricionais (PCN) para dietas orais em } \\
\text { hospitais? (2) Como ocorre o fluxo de informações no processo de prescrição de } \\
\text { dietas orais em hospitais? (3) Como o design da informação participa do fluxo de } \\
\text { informações no processo de prescrição de dietas orais em hospitais? }\end{array}$ \\
\hline $\begin{array}{l}\text { Domínios } \\
\text { da pesquisa }\end{array}$ & nutrição em hospitais; gestão hospitalar; design da informação \\
\hline Palavras-chave & nutrição; prescrição; dieta; hospital, fluxo; informação; design \\
\hline $\begin{array}{l}\text { Strings } \\
\text { de busca }\end{array}$ & $\begin{array}{l}\text { (flow AND information AND prescription AND diet AND hospita*) OR (prescription AND } \\
\text { diet AND nutrition) NOT enteral NOT parenteral; } \\
\text { (hospita AND diet AND prescription AND design) AND (flow OR information OR } \\
\text { nutrition) NOT enteral NOT parenteral. }\end{array}$ \\
\hline Bases & BVS; Lilacs; Scopus; Pubmed; Science Direct; Ebsco e Springer \\
\hline $\begin{array}{l}\text { Revistas } \\
\text { de Nutrição }\end{array}$ & $\begin{array}{l}\text { European Journal of Clinical Nutrition - Nature; Demetra, Rasbran, e The American } \\
\text { Journal of Clinical Nutrition (AJCN). }\end{array}$ \\
\hline $\begin{array}{l}\text { Revistas de } \\
\text { Gestão da } \\
\text { Informação }\end{array}$ & $\begin{array}{l}\text { Revistas Eletrônicas Puc/SP; Revista Economia e Gestão Puc/MG; Revista Gestão } \\
\text { Organizacional /Unochapecó; Navus - Revista de Gestão e Tecnologia SENAC/SC; } \\
\text { Revista de Gestão em Sistemas de Saúde; Revista Eletrônica de Sistemas de } \\
\text { Informação; Revista Eletrônica Gestão \& Saúde/UNB. }\end{array}$ \\
\hline $\begin{array}{l}\text { Revistas } \\
\text { de Design }\end{array}$ & $\begin{array}{l}\text { Design e Tecnologia; Estudos em Design; Infodesign; Strategic Design Research } \\
\text { Journal (Unisinos) e Information Design Journal (IDJ). }\end{array}$ \\
\hline $\begin{array}{l}\text { Critérios } \\
\text { de inclusão }\end{array}$ & $\begin{array}{l}\text { Artigos completos publicados e disponíveis integralmente nas bases científicas } \\
\text { buscadas; idiomas inglês e português; artigos que descrevam o processo de } \\
\text { prescrição de dietas orais em hospitais; artigos que discorram e apresentem o fluxo de } \\
\text { informações no processo de prescrição de dietas em hospitais; artigos que discorram } \\
\text { sobre o design de artefatos gráficos no processo de prescrição de dietas em hospitais }\end{array}$ \\
\hline $\begin{array}{l}\text { Critérios } \\
\text { de exclusão }\end{array}$ & $\begin{array}{l}\text { Artigos não disponíveis integralmente; artigos que não apresentem resumo/abstract; } \\
\text { artigos que abordem sobre o processo de prescrição de dietas enterais ou parenterais } \\
\text { em hospitais; artigos sobre câncer, diabetes, obesidade, nutrição infantil, CTI. }\end{array}$ \\
\hline
\end{tabular}

\section{Fase 2 da RBS: Processamento}

Mediante esse planejamento deu-se início a segunda fase, processamento. Para a configuração das buscas nas bases/revistas foi utilizado como apoio o software gratuito - StArt, (gerenciador de revisão sistemática de bibliografia), desenvolvido pelo Laboratório de Pesquisa em Engenharia de Software (LaPES) da Universidade Federal de São Carlos (UFSCar). Nesse software, o pesquisador configura os atributos do planejamento de entrada, na etapa protocolo, 
incluindo os arquivos resultantes da exportação das bases. Em seguida, na etapa execução, são definidos quais trabalhos serão aceitos ou rejeitados, de acordo com os critérios de inclusão e exclusão, somados à leitura dos títulos, palavras-chave e resumos. Logo após são selecionados àqueles trabalhos para leitura completa. Para a fase de saída da revisão, o StArt possibilita a geração de recursos de visualização de dados da revisão como gráficos e fluxogramas, segundo os critérios de classificação estabelecidos previamente.

A fase 3 da RBS - síntese e discussão dos resultados, será abordada na próxima seção.

\section{Resultados e discussão da RBS}

Após a condução das buscas e a exportação dos artigos localizados, deu-se início a fase de análise inicial dos resultados. A busca nas bases totalizou 234 artigos, entre esses 8 foram verificados como duplicados. Os 226 artigos restantes foram lidos os títulos e resumos. Entre esses foram excluídos 223 artigos que não contemplavam os critérios de inclusão, resultando em 3 artigos considerados como incluídos para a leitura completa. Os artigos selecionados foram publicados entre os anos de 2013 e 2020, na língua inglesa, e extraídos das bases Scopus e Science Direct. O quadro 2 resume os resultados das buscas nas bases perante os strings definidos, onde $\mathrm{R}$ são o número de artigos resultantes da busca e $\mathrm{S}$ àqueles que foram selecionados.

Quadro 2: Resumo das buscas nas bases. Fonte: elaborado pelas autoras.

1 ("flow" AND "information" AND "prescription" AND "diet" AND "hospita") OR ("prescription" AND "diet" AND "nutrition" AND "hospita") NOT "enteral" NOT "parenteral"

\begin{tabular}{|c|c|c|c|c|c|c|c|c|c|c|c|c|c|}
\hline \multicolumn{2}{|c|}{ BVS } & \multicolumn{2}{|c|}{ LILACS } & \multicolumn{2}{|c|}{ SCOPUS } & \multicolumn{2}{|c|}{ PUBMED } & \multicolumn{2}{|c|}{ Science Direct } & \multicolumn{2}{|c|}{ EBSCO } & \multicolumn{2}{|c|}{ Sringer Link } \\
\hline $\mathrm{R}$ & $S$ & $\mathrm{R}$ & S & $R$ & $\mathbf{s}$ & $\mathrm{R}$ & S & $\mathrm{R}$ & $\mathbf{s}$ & $\mathrm{R}$ & $\mathrm{S}$ & $\mathrm{R}$ & S \\
\hline 0 & 0 & 15 & 0 & 18 & 2 & 68 & 0 & 34 & 1 & 17 & 0 & 69 & 0 \\
\hline \multicolumn{14}{|c|}{2 ("hospita” AND "diet” AND "prescription" AND "design”) AND (“flow” OR “information” OR "nutrition") } \\
\hline \multicolumn{2}{|c|}{ BVS } & \multicolumn{2}{|c|}{ LILACS } & \multicolumn{2}{|c|}{ SCOPUS } & \multicolumn{2}{|c|}{ PUBMED } & \multicolumn{2}{|c|}{ Science Direct } & \multicolumn{2}{|c|}{ EBSCO } & \multicolumn{2}{|c|}{ Sringer Link } \\
\hline $\mathrm{R}$ & S & $\mathrm{R}$ & $s$ & $\mathrm{R}$ & $s$ & $\mathrm{R}$ & $s$ & $\mathrm{R}$ & S & $\mathrm{R}$ & $\mathrm{S}$ & $\mathrm{R}$ & S \\
\hline 0 & 0 & 0 & 0 & 0 & 0 & 7 & 0 & 1 & 0 & 5 & 0 & 0 & 0 \\
\hline \multicolumn{4}{|c|}{ Total de 234 artigos } & \multicolumn{4}{|c|}{ Duplicados: 8} & \multicolumn{4}{|c|}{ Incluídos: 3} & & \\
\hline
\end{tabular}

De modo geral, entre os estudos encontrados nas bases, a grande maioria $(n=96 \%)$ trata de assuntos estritamente relacionados à saúde e a nutrição. A busca nas revistas de nutrição, gestão da informação e design resultou em apenas um artigo que foi eliminado pelos critérios de exclusão. Após a finalização das buscas, foram incluídos apenas 3 artigos os quais foram lidos a fim de pontuar fatores que contribuem para a compreensão da administração de serviços nutricionais, e sua possível relação com o design da informação.

No primeiro artigo, Roberts (2013, p. 556) apresenta uma pesquisa acerca do "processo de implementação e os resultados de um programa de redação de pedidos de prescrição de 
nutricionistas em um grande centro médico urbano". Segundo a autora, "tradicionalmente, os nutricionistas registrados $(R D)$ não têm privilégios de redação de pedidos na maioria das instalações de atendimento ao paciente e dependem de médicos para implementar suas recomendações". Além disso, uma alta porcentagem de recomendações dos nutricionistas não são solicitadas. Nesse sentido, a autora reforça que a possibilidade de solicitação de "pedido por parte dos nutricionistas permite intervenções oportunas, melhores resultados, como melhor estado nutricional" (ROBERTS, 2013, p. 556). Nesse âmbito entende-se que os artefatos gráficos que participam da prática diária desses nutricionistas podem dificultar (e.g. deficiências na configuração gráfica dos artefatos) e interferir nas tomadas de decisão. Assim, a participação do design da informação para a promoção de melhorias no registro de informações em artefatos gráficos, pode contribuir para o trabalho dos nutricionistas que atuam como porta-voz das necessidades do paciente.

O segundo artigo dos autores Moya, D. et al. (2016, p. 316) discute sobre a desnutrição hospitalar, presente em cerca de $50 \%$ dos pacientes, e "associada ao aumento da morbidade, mortalidade e custo do atendimento". Os autores chamam a atenção para a "falta de alimentação ou terapia nutricional adequada aos pacientes. Práticas de jejum desnecessárias e o uso de prescrições nutricionais inadequadas resulta em subalimentação, que pode ser uma causa relacionada ou direta de desnutrição hospitalar"[...]. Na sequência, o artigo indica que sejam praticados os princípios bioéticos da medicina, como forma de: fornecer a cada paciente acesso a uma alimentação de qualidade; respeitar a autonomia dos pacientes sobre as opções de jejum e alimentação durante a hospitalização; promover o benefício ao paciente por meio de um cuidado nutricional ideal; e evitar as práticas de jejum e subalimentação, que prejudicam os pacientes e afetam seu bem-estar e cura. Com base nessas questões, reforça-se a importância dos artefatos que possuem complexidade textual (e.g. formulários e quadros), que podem induzir a problemas e falhas (e.g. falta de espaços horizontais e verticais destinados ao preenchimento) no momento do registro das informações, escritas à mão ou digitadas (Fontenele et al., 2019; Pena \& Melleiro, 2017; Quitério et al., 2016; Porterfield et al., 2014; Valadão et al., 2009; Reis et al., 2004).

No terceiro artigo publicado por Seguy, et al. (2020), o tema central é a desnutrição em idosos causada por condições patológicas, sociais, econômicas ou ambientais. Os autores argumentam que a adesão à suplementação nutricional oral pode diminuir o risco de hospitalização em idosos desnutridos sem custos extras com cuidados de saúde. Nesse sentido, os autores afirmam que "o manejo da desnutrição é baseado em aconselhamento dietético, assistência técnica ou humana na hora das refeições, fortificação de alimentos, adaptação de textura e prescrição de suplementos nutricionais orais densos em energia e proteína" (Seguy, et al., 2020, p. 1901). Considerando a necessidade do cuidado nutricional individual ao paciente, percebe-se que a relação com os artefatos gráficos na prescrição dietética pode sofrer interferências perante o modo como os componentes informacionais se apresentam e se organizam. Desse modo, a organização e distribuição desses componentes podem interferir, confundir e/ou dificultar tanto o preenchimento como a 
localização de dados pela equipe de saúde, prejudicando o suprimento das necessidades nutricionais do paciente internado.

Diante das questões observadas nesses artigos, verifica-se que através dos aspectos e princípios do design da informação (Pettersson, 2012b; Waarde, 2004; Horn, 1998; Schriver, 1997) é possível contribuir mediante a compreensão, estruturação e formatação dos artefatos gráficos essenciais às atividades de cada profissional. Desse modo é possível facilitar a visualização, localização e preenchimento dos artefatos, de forma que as informações essenciais ao PCN possam fluir com mais precisão, confiabilidade, relevância e clareza (Audy; et al., 2005, p. 95)

Porém, ao final dessa revisão bibliográfica não foi localizado qualquer artigo que mencione sobre: o processo de cuidados nutricionais em hospitais, o fluxo de informações no processo de prescrição de dietas orais em hospitais, além de aspectos do design da informação dos artefatos gráficos que atuam no processo de prescrição de dietas orais em hospitais.

Percebe-se, então, uma escassez de publicações, nacionais e internacionais, sobre o design da informação no âmbito da saúde, especificamente no campo da nutrição hospitalar, que estejam indexados nas principais bases de dados.

Mediante esse resultado, enfatiza-se que possíveis problemas ocasionados por artefatos gráficos que participam do PCN não estão sendo considerados como relevantes. Porém, o artigo em curso ressalta a importância desse campo de atuação para o design de informação assim como para a nutrição hospitalar.

\section{Considerações finais}

Este artigo apresentou uma revisão bibliográfica sistemática com o intuito de pontuar campos de conhecimento no âmbito da assistência nutricional, processo, fluxo de informações em hospitais, assim como design da informação direcionado a artefatos gráficos que participem desse contexto.

O método de revisão bibliográfica desenvolvido pelo modelo de Levi e Ellis (2006) que utiliza a abordagem "entrada-processamento-saída", permitiu a descrição, organização e sistematização das buscas nas bases e revistas especializadas, com profundidade. Contudo é oportuno esclarecer que a extração dos artigos selecionados, na maioria das bases de dados, só foi possível mediante o acesso ao Portal de Periódicos Capes.

A utilização do software StArt (LaPES - UFSCar) possibilitou o gerenciamento de seis das sete bases pesquisadas na RBS. Porém, houve uma dificuldade na utilização do aplicativo por não reconhecer arquivos indexados em formato CSV disponibilizado por uma das bases. Os artigos que estavam nesse formato foram selecionados no modo manual e acrescidos ao cômputo geral da pesquisa.

Outra dificuldade no desenvolvimento dessa pesquisa deu-se pela lacuna identificada sobre artigos científicos relativos ao design da informação atuando no campo da nutrição hospitalar. A técnica da RBS demonstrou que para a área da saúde o acesso aos artigos é abrangente e 
disponível. Contudo foi verificado que no âmbito do design da informação, os artigos científicos não estão indexados nas principais bases de dados. Assim, a utilização da técnica da RBS não foi suficiente para apreender os campos de estudo direcionados a artefatos gráficos, relacionados a assistência nutricional, processo e fluxo de informações em hospitais. Além disso, outros canais de divulgação científica do design da informação em saúde, como anais de eventos, livros e teses, não foram encontrados nas bases de buscas.

Diante dessa realidade, destaca-se, oportunidade de estudos em design da informação no âmbito do desenvolvimento de artefatos gráficos e informacionais na área de assistência nutricional hospitalar, que contribuirão tanto para a pesquisa como para a prática do design da informação.

\section{Agradecimentos}

Agradecemos à Coordenação de Aperfeiçoamento de Pessoal de Nível Superior (CAPES), e ao Programa de Pós-graduação em Design da Universidade Federal do Paraná (PPGDesign UFPR) por viabilizarem o desenvolvimento dessa pesquisa.

\section{Referências}

American Dietetic Association. (1994). Identifying patients at risk: ADA's definitions for nutrition screening and nutritional assessment. J Am Diet Association, v. 94, n. 8, p. 838-839,.

Audy, J. L. N.; Andrade, G. K. de.; Cidral, A. (2005). Fundamentos de sistemas de informação. Porto Alegre: Bookman.

Brito, K. M. (2015). Riscos à saúde dos trabalhadores em unidade de alimentação e nutrição de um hospital universitário. (Dissertação de Mestrado em Gestão de Processos Institucionais. Universidade Federal do Rio Grande do Norte).

Ceriolli, D. et al. (2015). Fluxo informacional e gestão hospitalar: um estudo de caso no hospital e maternidade municipal de Pimenta Bueno-RO. Revista de Administração Hospitalar e Inovação em Saúde, v. 12, n. 3, p. 76-93.

Cunha, F. J. A. P.; Silva, H. P. da. (2005). Informação e comunicação no contexto dos hospitais. Intercom, p. 1-15.

De Seta, M. H. et al. (2010). Cuidado nutricional em hospitais públicos de quatro estados brasileiros: contribuições da avaliação em saúde à vigilância sanitária de serviços. Ciência e Saúde Coletiva, v. 15, n. SUPPL. 3, p. 3413-3422.

Fontenele, R. M. et al. (2019). Comunicação ineficaz e suas consequências para o paciente grave. Revista Recien - Revista Científica de Enfermagem, v. 9, n. 27, p. 117.

Greef, A. C.; Freitas, M. do C.; Romanel, F. (2012). Lean office: operação, gerenciamento e tecnologias. São Paulo: Atlas.

Hakel-Smith, N.; Lewis, N. M. (2004). A standardized nutrition care process and language are essential components of a conceptual model to guide and document nutrition care and patient outcomes. Journal of the American Dietetic Association, v. 104, n. 12, p. 1878-1884. 
Horn, R. (1998). Visual language: global communication for the 21st century. Bainbridgee Island, WA: MacroVU, Inc.

Howard, P. et al. (2006). Managing the patient journey through enteral nutritional care. Clinical Nutrition, v. 25, n. 2, p. 187-195.

Levy, Y.; Ellis, T.J. (2006). A system approach to conduct an effective literature review in support of Information systems research. Informing Science Journal, v.9, p.181-212.

Maculevicius, J.; Dias, M. C. (2006). Dietas orais hospitalares. In: Waitzberg, D. (Ed.). Nutrição oral, enteral e parenteral na prática clínica. $3^{\mathrm{a}}$ ed. São Paulo: Editora Atheneu, p. 465-480.

Mowe, M. et al. (2006). Nutritional routines and attitudes among doctors and nurses in Scandinavia: a questionnaire based survey. Clinical Nutrition, v. 25, n. 3, p. 524-532.

Moya, D. et al. (2016). Hospital Malnutrition Related to Fasting and Underfeeding: Is It an Ethical Issue? Nutrition in Clinical Practice, v. 31, n. 3, p. 316-324.

Pedroso, C. G. T. (2007). Cuidado alimentar e nutricional ao paciente hospitalizado: elementos para a construção de um modelo fundamentado na humanização. Orientadora: Anete Araújo de Sousa. Dissertação (Mestrado) Programa de Pós-Graduação em Nutrição. Universidade Federal de Santa Catarina.

Pedroso, C. G. T.; Sousa, A. A. de; Salles, R. K. DE. (2011). Cuidado nutricional hospitalar: percepção de nutricionistas para atendimento humanizado. Ciência e Saúde Coletiva, v. 1, p. 1155-1162.

Pena, M. M.; Melleiro, M. M. (2017). O método de análise de causa raiz para a investigação de eventos adversos. Rev. Enferm. UFPE on line, v. 11, n. supl.12, p. 5297-5304.

Peruzzi, L. M. et al. (2019). Passagem de plantão na atenção hospitalar. Rev. Enferm. UFPE on line, v. 13, n. 4, p. 989-996.

Pettersson, R. (2012b). Introduction to message design. Journal of Visual Literacy, v. 31, n. 2, p. 93-104.

Porterfield, A.; Engelbert, K.; Coustasse, A. (2014). Electronic prescribing: improving the efficiency and accuracy of prescribing in the ambulatory care setting. Perspectives in Health Information Management, v. 11, p.1-13.

Quitério, L. M. et al. (2016). Eventos adversos por falhas de comunicação em unidades de terapia intensiva. Espacios, v. 37, n. 30, p. 1-10.

Reis, C. V. dos; et al. (2004). Modelo de implantação da prescrição informatizada de terapia nutricional em um serviço de nutrição hospitalar. Telemedicina Unifesp.

Roberts, S. R. (2013). Improving patient outcomes through registered dietitian order writing. Nutrition in Clinical Practice, v. 28, n. 5, p. 556-565.

Schriver, K. A. (1997). Dynamics in document design. USA: John Wiley \& Sons.

Seguy, D. et al. (2020). Compliance to oral nutritional supplementation decreases the risk of hospitalisation in malnourished older adults without extra health care cost: Prospective observational cohort study. Clinical Nutrition, v. 39, n. 6, p. 1900-1907.

Sless, D. (1996). Better information presentation: satisfying customers? Visible Language, v. 30 , n. 3 , p. 246-267. 
Valadão, A. F. et al. (2009). Prescrição médica: um foco nos erros de prescrição. Rev Bras Farm, v. 90, n. 4, p. 340-343.

Waarde, K. Van Der. (2004). The graphic presentation of patient package inserts. In: Zwaga, H.; Boersema, T.; Hoonhout, H. (Eds.) Visual information for everyday use: design and research perspectives. London: Taylor \& Francis Ltd. 